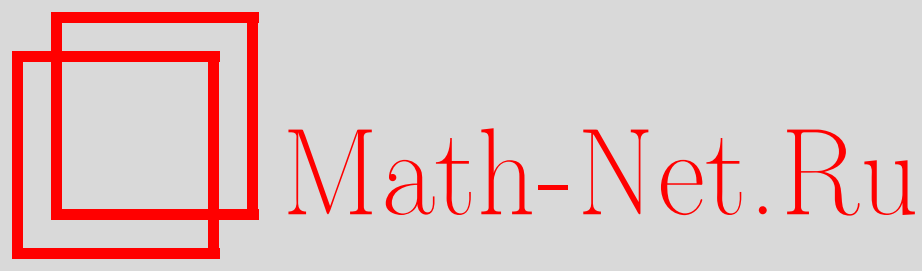

Г. Г. Козлов, Вычисление степени локализации в смысле критерия Андерсона для одномерной диагонально разупорядоченной системы, ТМФ, 2010, том 162, номер 2, 285-303

DOI: https://doi.org/10.4213/tmf6471

Использование Общероссийского математического портала Math-Net.Ru подразумевает, что вы прочитали и согласны с пользовательским соглашением http: //www.mathnet.ru/rus/agreement

Параметры загрузки:

IP : 54.92 .164 .108

26 апреля 2023 г., 18:26:31

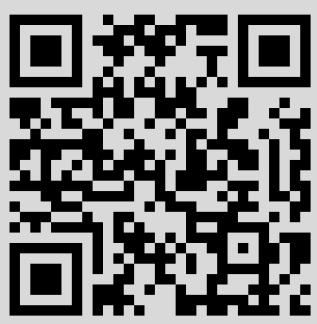




\title{
ВЫЧИСЛЕНИЕ СТЕПЕНИ ЛОКАЛИЗАЦИИ В СМЫСЛЕ КРИТЕРИЯ АНДЕРСОНА ДЛЯ ОДНОМЕРНОЙ ДИАГОНАЛЬНО РАЗУПОРЯДОЧЕННОЙ СИСТЕМЫ
}

\begin{abstract}
Для одномерной диагонально разупорядоченной полубесконечной цепочки рассмотрена задача о предельном при $t \rightarrow \infty$ значении средней плотности возбуждения $D$ на крайнем узле цепочки при условии, что при $t=0$ возбуждение было локализовано на этом узле. Для бинарно-разупорядоченной цепочки получено выражение для $D$, точное в пределе малой концентрации дефектов и при произвольной их энергии. При этом $D$ демонстрирует неаналитическую зависимость от энергии. Получено выражение для $D$ при произвольном малом диагональном беспорядке. Рассчитан относительный вклад в $D$ состояний с заданной энергией. Все полученные результаты находятся в согласии с данными компьютерного моделирования.
\end{abstract}

Ключевые слова: разупорядоченная система, случайная матрица, локализация состояний, критерий Андерсона.

\section{1. ВВЕДЕНИЕ, ПОСТАНОВКА ЗАДАЧИ И ОСНОВНЫЕ РЕЗУЛЬТАТЫ}

Математические модели современной физики разупорядоченных систем можно разделить на два класса - непрерывные и дискретные. К непрерывным относятся модели, в которых изучается уравнение Шредингера $(-\Delta+v(r)) \psi=E \psi$ со случайным потенциалом $v(r)$, к дискретным - модели, в которых исследуется случайная матрица гамильтониана разупорядоченной системы. Несмотря на сходство, модели этих двух классов могут потребовать существенно разных методов анализа по следующей причине. Как известно [1], одним из важнейших свойств пространственно однородных разупорядоченных систем является возможность появления в них локализованных состояний. Волновые функции этих состояний существенно отличны от нуля в областях, средний размер которых, оставаясь конечным, перестает зависеть от объема разупорядоченной системы, когда этот объем стремится к бесконечности. Для непрерывных моделей бесконечного объема критерием локализации,

* Государственный оптический институт им. С. И. Вавилова, Санкт-Петербург, Россия. E-mail: gkozlov@photonics.phys.spbu.ru 
по-видимому, может служить разделение энергетического спектра на дискретную и непрерывную части, причем локализованными считаются интегрируемые с квадратом состояния дискретной части спектра. Энергетический спектр дискретных моделей, будучи спектром случайной матрицы, всегда дискретен, поэтому для характеризации состояний в этом случае необходимо применение других критериев, одним из которых является критерий Андерсона [1], [2].

Наиболее глубокий теоретический анализ возможен для одномерных моделей разупорядоченных систем, которые мы и будем иметь в виду ниже. Для одномерных непрерывных моделей доказано, что уравнение Шредингера со случайным однородным потенциалом достаточно общего вида имеет только дискретный спектр, что соответствует локализованному характеру всех состояний при сколь угодно малом беспорядке [1], [3], [4]. Перенесение этого утверждения на дискретные модели, на наш взгляд, не очевидно по причине полной дискретности спектра таких моделей даже при отсутствии беспорядка, когда все состояния заведомо делокализованы. Исследование дискретных одномерных разупорядоченных моделей представляется не менее актуальным, чем непрерывных, поскольку разупорядоченность целого ряда реальных физических систем (квантовые сверхрешетки, Ј-агрегаты, оптические волокна, многослойные брэгговские отражатели, квазиодномерные цепочки в трехмерных системах [5], [6]) описывается дискретными моделями. В предлагаемой работе для простейшей диагонально разупорядоченной цепочки выполнено исследование характера собственных состояний в смысле критерия Андерсона, который, обладая простым физическим смыслом, может быть применен как к дискретным, так и к непрерывным моделям.

Перейдем к постановке задачи. Рассмотрим одномерную дискретную модель диагонально разупорядоченной системы, для которой матрица гамильтониана Н имеет следующие элементы:

$$
H_{r, r^{\prime}}=\delta_{r, r^{\prime}} \varepsilon_{r}+\delta_{r, r^{\prime}+1}+\delta_{r, r^{\prime}-1}, \quad r, r^{\prime}=1, \ldots, N
$$

Такой гамильтониан описывает в приближении ближайших соседей экситон Френкеля в цепочке, состоящей из $N$ двухуровневых атомов. Расщепление $\varepsilon_{r}$ двухуровневого атома с номером $r$ в этой модели является случайной величиной, плотность распределения $P$ которой предполагается известной. Равные единице недиагональные элементы в (1) определяют единицу измерения энергии. Всюду ниже мы будем подразумевать термодинамический предел $N \rightarrow \infty$.

Для такой модели рассмотрим следующую задачу. Пусть при $t=0$ крайний атом $(r=N)$ был возбужден; требуется найти вероятность $D$ того, что этот атом останется в возбужденном состояние при $t \rightarrow \infty$. Математически это означает, что начальное состояние системы описывается волновой функцией (вектор-столбцом) $\boldsymbol{\Psi}(0)$ с компонентами $\Psi_{r}(0)=\delta_{r, N}$, и требуется найти $D=\left.\left\langle\left|\Psi_{N}(t)\right|^{2}\right\rangle\right|_{t \rightarrow \infty} ;$ здесь угловые скобки обозначают усреднение по реализациям случайных расщеплений $\varepsilon_{r}$, $r=1, \ldots, N$. Зависимость волновой функции системы от времени определяется следующим образом: $\boldsymbol{\Psi}(t)=e^{i \mathbf{H} t} \boldsymbol{\Psi}(0)$. Отсюда вытекает, что интересующая нас величина $D$ может быть выражена через собственные векторы $\boldsymbol{\Psi}^{\lambda}$ и собственные 
значения $E_{\lambda}, \lambda=1, \ldots, N$, матрицы (1) следующим образом:

$$
D=\left.\left\langle\left|\Psi_{N}(t)\right|^{2}\right\rangle\right|_{t \rightarrow \infty}=\lim _{t \rightarrow \infty}\left\langle\sum_{\lambda, \lambda^{\prime}}\left|\Psi_{N}^{\lambda}\right|^{2}\left|\Psi_{N}^{\lambda^{\prime}}\right|^{2} e^{i\left(E_{\lambda}-E_{\lambda^{\prime}}\right) t}\right\rangle=\left\langle\sum_{\lambda}\left|\Psi_{N}^{\lambda}\right|^{4}\right\rangle
$$

Сходные величины анализируются при численном исследовании разупорядоченных цепочек, например, в работе [7]. Относительно величины $D$ можно сделать следующие качественные заключения. Допустим, что все собственные функции гамильтониана (1) делокализованы в том смысле, что их амплитуда примерно одинакова в любой области цепочки. Квадрат амплитуды таких функций в крайнем узле оценивается как $\left|\Psi_{N}^{\lambda}\right|^{2} \sim 1 / N$, причем все $N$ собственных функций вносят в (2) примерно одинаковый вклад: $\left|\Psi_{N}^{\lambda}\right|^{4} \sim 1 / N^{2}$. Следовательно, если состояния (1) делокализованы в указанном выше смысле, то в термодинамическом пределе $D \sim 1 / N \rightarrow 0$. Рассмотрим теперь ситуацию, когда среди собственных функций (1) есть локализованные в том смысле, что их амплитуда существенно отличается от нуля лишь в некоторой области цепочки, причем размер этой области не зависит от $N$ при $N \rightarrow \infty$. Вклад функций такого типа в (2) не будет зависеть от $N$, поскольку он определяется лишь теми из них, которые имеют существенно отличную от нуля амплитуду на крайнем узле. Тогда величина $D$ остается конечной в термодинамическом пределе. Критерий Андерсона вводится на основе приведенных рассуждений следующим образом: если величина $D$ конечна, то среди собственных функиий гамильтониана (1) есть локализованные в смысле критерия Андерсона.

Для того чтобы судить о степени локализации собственных векторов гамильтониана (1) в спектральном интервале $[U, U+d U]$, введем функцию “участия" $W(U)$, которую определим следующим соотношением:

$$
W(U) d U=\left\langle\sum_{E_{\lambda} \in[U, U+d U]}\left|\Psi_{N}^{\lambda}\right|^{4}\right\rangle
$$

Очевидно, $D=\int W(U) d U$. Рассуждения, аналогичные приведенным выше, показывают, что если все состояния в интервале $[U, U+d U]$ делокализованы, то $W(U)=0$. В противном случае $W(U)$ отлична от нуля. Кроме того, функция (3) дает количественную информацию о средней величине собственных векторов случайной матрицы (1) на крайнем узле в спектральном интервале $[U, U+d U]$. Фантастические возможности современных персональных компьютеров позволяют за обозримое время проводить диагонализацию матриц (1) и в таком численном эксперименте "наблюдать" величины $(2)$ и (3) при $N \gtrsim 1000$. Основной задачей предлагаемой работы является теоретический расчет этих величин.

В работе получены следующие основные результаты.

1. Построена теория возмущений для вычисления совместной плотности распределения опережающей и запаздывающей функций Грина гамильтониана (1). 
2. Для бинарно разупорядоченной одномерной системы, описывающейся гамильтонианом (1), в котором атомные расщепления $\varepsilon_{r}$ с вероятностью $1-c$ равны нулю и с вероятностью $c$ равны $\varepsilon, 0<c<1$, получены следующие выражения для величины $D$ и функции $W(U)$ :

$$
\begin{aligned}
D= & \frac{c}{4 \pi} \int_{-2}^{2}\left(4-U^{2}\right)^{3 / 2} \ln \left(\frac{\varepsilon^{2}}{4-U^{2}}+1\right) d U+ \\
& +c \Theta(|\varepsilon|-1)\left(\frac{\varepsilon^{2}-1}{\varepsilon^{2}}\right)^{2}+O\left(c^{2}\right), \\
W(U)= & \frac{c}{4 \pi} \Theta(2-|U|)\left(4-U^{2}\right)^{3 / 2} \ln \left(\frac{\varepsilon^{2}}{4-U^{2}}+1\right)+ \\
& +c \Theta(|\varepsilon|-1)\left(\frac{\varepsilon^{2}-1}{\varepsilon^{2}}\right)^{2} \delta\left(U-\frac{\varepsilon^{2}+1}{\varepsilon}\right)+O\left(c^{2}\right) .
\end{aligned}
$$

Неаналитичность величины (4) как функции $\varepsilon$ связана с появлением при $|\varepsilon|>1$ краевого состояния с энергией $U_{0}=\left(\varepsilon^{2}+1\right) / \varepsilon$.

3. Для широкого класса разупорядоченных систем, описывающихся гамильтонианом (1), в котором случайные атомные расщепления $\varepsilon_{r}$ имеют плотность распределения вида $P_{\Delta}(x)=p(x / \Delta) / \Delta$, где

$$
p(x)>0, \quad \int p(x) d x=1, \quad \int p(x) x d x=0, \quad \int p(x) x^{2} d x=M_{2},
$$

получены следующие выражения для величины $D$ и функции $W(U)$ :

$$
D=\frac{\Delta^{2} M_{2}}{2}+O\left(\Delta^{3}\right), \quad W(U)=\Theta(2-|U|) \frac{\Delta^{2} M_{2}}{4 \pi} \sqrt{4-U^{2}}+O\left(\Delta^{3}\right) .
$$

Все полученные в работе результаты сравниваются с результатами компьютерного моделирования, которое показывает, что при $D<0.1$ значащие части формул (4)-(6) дают погрешность меньше 10\%. При этом степень разупорядоченности системы может быть довольно большой: например, значащие части формул (6) оказываются применимыми, если $\varepsilon_{r}$ равномерно распределено в интервале [-0.25, 0.25].

\section{2. СТАТИСТИКА ФУНКЦИЙ ГРИНА}

Нетрудно показать, что на краевом узле средний квадрат модуля волновой функции $\left\langle\left|\Psi_{N}(t)\right|^{2}\right\rangle$ (с которым интересующая нас величина (2) связана как $D=$ $\left.=\left\langle\left|\Psi_{N}(\infty)\right|^{2}\right\rangle\right)$ может быть вычислен следующим образом:

$$
\left\langle\left|\Psi_{N}(t)\right|^{2}\right\rangle=\lim _{V_{1,2} \rightarrow+0} \frac{1}{4 \pi^{2}} \int e^{i\left(U_{1}-U_{2}\right) t}\left\langle\gamma\left(U_{1}-i V_{1}\right) \gamma\left(U_{2}+i V_{2}\right)\right\rangle d U_{1} d U_{2}
$$


где $\gamma(\Omega)$ - краевая функция Грина (КФГ) для гамильтониана (1),

$$
\gamma(\Omega) \equiv \sum_{\lambda} \frac{\left|\Psi_{N}^{\lambda}\right|^{2}}{\Omega-E_{\lambda}} .
$$

Для вычисления входящего в (7) среднего значения произведения двух функций Грина необходимо знать их совместную функцию распределения. Получим уравнение для этой функции, обобщив метод Дайсона [1], [8]. Обозначим через $\gamma\left(\Omega_{i}\right), i=1,2$, краевые функции Грина (8) цепочки при комплексных энергиях $\Omega_{1} \equiv U_{1}-i V_{1}$ и $\Omega_{2} \equiv U_{2}+i V_{2}$ и добавим к цепочке еще один атом с расщеплением $\varepsilon$. Тогда, как показано в работах [1], [8], КФГ $\widetilde{\gamma}(\Omega)$ цепочки с добавленным атомом связана с КФГ исходной цепочки соотношением

$$
\widetilde{\gamma}(\Omega)=\frac{1}{\Omega-\varepsilon-\gamma(\Omega)} .
$$

Для описания краевых функций Грина $\gamma\left(\Omega_{i}\right), i=1,2$, исходной цепочки введем плотность распределения $\eta$ такую, что величина $\eta\left(x_{1}, y_{1}, x_{2}, y_{2}\right) d x_{1} d y_{1} d x_{2} d y_{2}$ есть вероятность того, что $\operatorname{Re} \gamma\left(\Omega_{i}\right) \in\left[x_{i}, x_{i}+d x_{i}\right]$ и $\operatorname{Im} \gamma\left(\Omega_{i}\right) \in\left[y_{i}, y_{i}+d y_{i}\right], i=1,2$. Обозначим через $\widetilde{\eta}$ аналогичную функцию для цепочки с добавленным атомом. Соотношение (9) позволяет выразить $\widetilde{\eta}$ через $\eta$ и плотность распределения атомных расщеплений $P(\varepsilon)$ :

$$
\begin{aligned}
& \tilde{\eta}\left(\tilde{x}_{1}, \tilde{y}_{1}, \tilde{x}_{2}, \tilde{y}_{2}\right)=\int \delta\left(\tilde{x}_{1}-\operatorname{Re} \frac{1}{\Omega_{1}-\varepsilon-x_{1}-i y_{1}}\right) \delta\left(\tilde{y}_{1}-\operatorname{Im} \frac{1}{\Omega_{1}-\varepsilon-x_{1}-i y_{1}}\right) \times \\
& \quad \times \delta\left(\tilde{x}_{2}-\operatorname{Re} \frac{1}{\Omega_{2}-\varepsilon-x_{2}-i y_{2}}\right) \delta\left(\tilde{y}_{2}-\operatorname{Im} \frac{1}{\Omega_{2}-\varepsilon-x_{2}-i y_{2}}\right) \times \\
& \quad \times \eta\left(x_{1}, y_{1}, x_{2}, y_{2}\right) P(\varepsilon) d x_{1} d y_{1} d x_{2} d y_{2} d \varepsilon .
\end{aligned}
$$

В термодинамическом пределе $N \rightarrow \infty$ должно быть $\eta=\widetilde{\eta}$. Вычисляя интегралы с $\delta$-функциями в (10), получаем для стационарной функции $\eta$ следующее уравнение:

$$
\begin{aligned}
& \left(x_{1}^{2}+y_{1}^{2}\right)^{2}\left(x_{2}^{2}+y_{2}^{2}\right)^{2} \eta\left(x_{1}, y_{1}, x_{2}, y_{2}\right)= \\
& \quad=\int \eta\left(U_{1}-\varepsilon-\frac{x_{1}}{x_{1}^{2}+y_{1}^{2}},-V_{1}+\frac{y_{1}}{x_{1}^{2}+y_{1}^{2}}, U_{2}-\varepsilon-\frac{x_{2}}{x_{2}^{2}+y_{2}^{2}}, V_{2}+\frac{y_{2}}{x_{2}^{2}+y_{2}^{2}}\right) P(\varepsilon) d \varepsilon .
\end{aligned}
$$

При помощи функции $\eta$ входящее в (7) среднее произведение опережающей и запаздывающей функций Грина можно представить как сумму четырех слагаемых:

$$
\begin{aligned}
\left\langle\gamma\left(\Omega_{1}\right) \gamma\left(\Omega_{2}\right)\right\rangle & =\int \eta\left(x_{1} y_{1} x_{2} y_{2}\right)\left[x_{1} x_{2}-y_{1} y_{2}+i\left(y_{1} x_{2}+y_{2} x_{1}\right)\right] d x_{1} d x_{2} d y_{1} d y_{2} \equiv \\
& \equiv\left\langle x_{1} x_{2}\right\rangle-\left\langle y_{1} y_{2}\right\rangle+i\left\langle y_{1} x_{2}\right\rangle+i\left\langle y_{2} x_{1}\right\rangle .
\end{aligned}
$$


Используя выражение (8), нетрудно убедиться в том, что эти слагаемые можно записать в представлении гамильтониана (1) следующим образом:

$$
\begin{aligned}
\left\langle x_{1} x_{2}\right\rangle & =\left\langle\sum_{\lambda, \lambda^{\prime}} \frac{\left|\Psi_{N}^{\lambda}\right|^{2}\left|\Psi_{N}^{\lambda^{\prime}}\right|^{2}\left(U_{1}-E_{\lambda}\right)\left(U_{2}-E_{\lambda^{\prime}}\right)}{\left[\left(U_{1}-E_{\lambda}\right)^{2}+V_{1}^{2}\right]\left[\left(U_{2}-E_{\lambda^{\prime}}\right)^{2}+V_{2}^{2}\right]}\right\rangle, \\
-\left\langle y_{1} y_{2}\right\rangle & =\left\langle\sum_{\lambda, \lambda^{\prime}} \frac{\left|\Psi_{N}^{\lambda}\right|^{2}\left|\Psi_{N}^{\lambda^{\prime}}\right|^{2} V_{1} V_{2}}{\left[\left(U_{1}-E_{\lambda}\right)^{2}+V_{1}^{2}\right]\left[\left(U_{2}-E_{\lambda^{\prime}}\right)^{2}+V_{2}^{2}\right]}\right\rangle, \\
i\left\langle y_{1} x_{2}\right\rangle & =i\left\langle\sum_{\lambda, \lambda^{\prime}} \frac{\left|\Psi_{N}^{\lambda}\right|^{2}\left|\Psi_{N}^{\lambda^{\prime}}\right|^{2} V_{1}\left(U_{2}-E_{\lambda^{\prime}}\right)}{\left[\left(U_{1}-E_{\lambda}\right)^{2}+V_{1}^{2}\right]\left[\left(U_{2}-E_{\lambda^{\prime}}\right)^{2}+V_{2}^{2}\right]}\right\rangle, \\
i\left\langle x_{1} y_{2}\right\rangle & =-i\left\langle\sum_{\lambda, \lambda^{\prime}} \frac{\left|\Psi_{N}^{\lambda}\right|^{2}\left|\Psi_{N}^{\lambda^{\prime}}\right|^{2} V_{2}\left(U_{1}-E_{\lambda}\right)}{\left[\left(U_{1}-E_{\lambda}\right)^{2}+V_{1}^{2}\right]\left[\left(U_{2}-E_{\lambda^{\prime}}\right)^{2}+V_{2}^{2}\right]}\right\rangle .
\end{aligned}
$$

Каждому из этих слагаемых соответствует вклад в $\left\langle\left|\Psi_{N}(t)\right|^{2}\right\rangle$ :

$$
\left\langle\left|\Psi_{N}(t)\right|^{2}\right\rangle=\Delta_{\left\langle x_{1} x_{2}\right\rangle}+\Delta_{\left\langle y_{1} y_{2}\right\rangle}+\Delta_{\left\langle y_{1} x_{2}\right\rangle}+\Delta_{\left\langle x_{1} y_{2}\right\rangle} .
$$

Для примера приведем вычисление вклада $\Delta_{\left\langle y_{2} x_{1}\right\rangle}$ :

$$
\begin{aligned}
\Delta_{\left\langle y_{2} x_{1}\right\rangle}= & \frac{i}{4 \pi^{2}} \lim _{V_{1,2} \rightarrow+0} \int e^{i\left(U_{1}-U_{2}\right) t}\left\langle y_{2} x_{1}\right\rangle d U_{1} d U_{2}=-\frac{i}{4 \pi^{2}} \lim _{V_{1,2} \rightarrow+0} \int e^{i\left(U_{1}-U_{2}\right) t} \times \\
& \times\left\langle\sum_{\lambda, \lambda^{\prime}} \frac{\left|\Psi_{N}^{\lambda}\right|^{2}\left|\Psi_{N}^{\lambda^{\prime}}\right|^{2} V_{2}\left(U_{1}-E_{\lambda}\right)}{\left[\left(U_{1}-E_{\lambda}\right)^{2}+V_{1}^{2}\right]\left[\left(U_{2}-E_{\lambda^{\prime}}\right)^{2}+V_{2}^{2}\right]}\right\rangle d U_{1} d U_{2}= \\
= & \frac{1}{4}\left\langle\sum_{\lambda, \lambda^{\prime}}\left|\Psi_{N}^{\lambda}\right|^{2}\left|\Psi_{N}^{\lambda^{\prime}}\right|^{2} e^{i\left(E_{\lambda}-E_{\lambda^{\prime}}\right) t}\right\rangle .
\end{aligned}
$$

Аналогичные вычисления показывают, что все четыре слагаемых в $\left\langle\left|\Psi_{N}(t)\right|^{2}\right\rangle$ в формуле (14) равны между собой: $\Delta_{\left\langle x_{1} x_{2}\right\rangle}=\Delta_{\left\langle y_{1} y_{2}\right\rangle}=\Delta_{\left\langle y_{1} x_{2}\right\rangle}=\Delta_{\left\langle x_{1} y_{2}\right\rangle}$, следовательно,

$$
\left\langle\left|\Psi_{N}(t)\right|^{2}\right\rangle=4 \Delta_{\left\langle y_{2} x_{1}\right\rangle}
$$

Сделаем следующее важное для дальнейшего замечание. Допустим, что интегрирование по $U_{1,2}$ в формуле (15) мы проводим только по небольшой области $U_{1,2} \in[U, U+d U]$ и интересуемся поведением вклада $\Delta_{\left\langle y_{2} x_{1}\right\rangle}$ при $t \rightarrow \infty$. В этом случае сумма в правой части равенства (15) будет содержать только состояния с энергиями $E_{\lambda} \in[U, U+d U]$. Отсюда видно, что такое ограничение области интегрирования позволяет вычислять функцию “участия" (3).

Таким образом, задача сводится к решению уравнения (11) для функции совместной плотности вероятности $\eta$. Тот факт, что при вычислениях вкладов (13) подразумевается предельный переход $V_{1,2} \rightarrow+0$, можно использовать для сведения задачи к исследованию существенно более простого уравнения, чем (11). Для этого заметим, что если $V_{1}=V_{2}=0$, то решение уравнения (11) можно представить в виде

$$
\left.\eta\left(x_{1}, y_{1}, x_{2}, y_{2}\right)\right|_{V_{1,2}=0}=\delta\left(y_{1}\right) \delta\left(y_{2}\right) \rho\left(x_{1}, x_{2}\right)
$$


где функция $\rho\left(x_{1}, x_{2}\right)$ удовлетворяет следующему уравнению:

$$
x_{1}^{2} x_{2}^{2} \rho\left(x_{1}, x_{2}\right)=\int P(\varepsilon) \rho\left(U_{1}-\varepsilon-\frac{1}{x_{1}}, U_{2}-\varepsilon-\frac{1}{x_{2}}\right) d \varepsilon .
$$

Теперь выполним расчет величины $\left\langle y_{2} x_{1}\right\rangle$, учитывая, что при предельно малых $V_{1,2}$ решение уравнения (11) стремится к (17). Поскольку функция $\eta$ удовлетворяет уравнению (11), для искомого среднего $\left\langle y_{2} x_{1}\right\rangle$ можно написать следующее выражение:

$$
\begin{aligned}
& \left\langle y_{2} x_{1}\right\rangle=\int \eta\left(x_{1} y_{1} x_{2} y_{2}\right) y_{2} x_{1} d x_{1} d x_{2} d y_{1} d y_{2}=\int \frac{y_{2} x_{1} d x_{1} d x_{2} d y_{1} d y_{2} d \varepsilon}{\left(x_{1}^{2}+y_{1}^{2}\right)^{2}\left(x_{2}^{2}+y_{2}^{2}\right)^{2}} \times \\
& \quad \times \eta\left(U_{1}-\varepsilon-\frac{x_{1}}{x_{1}^{2}+y_{1}^{2}},-V_{1}+\frac{y_{1}}{x_{1}^{2}+y_{1}^{2}}, U_{2}-\varepsilon-\frac{x_{2}}{x_{2}^{2}+y_{2}^{2}}, V_{2}+\frac{y_{2}}{x_{2}^{2}+y_{2}^{2}}\right) P(\varepsilon) .
\end{aligned}
$$

Делая замену переменных

$$
-\frac{x_{k}}{y_{k}^{2}+x_{k}^{2}} \mapsto x_{k}, \quad \frac{y_{k}}{y_{k}^{2}+x_{k}^{2}} \mapsto y_{k}, \quad k=1,2
$$

и рассчитывая соответствующие якобианы, можно переписать правую часть (19) в виде

$-\int \eta\left(U_{1}-\varepsilon+x_{1}, y_{1}-V_{1}, U_{2}-\varepsilon+x_{2}, V_{2}+y_{2}\right) \frac{x_{1}}{y_{1}^{2}+x_{1}^{2}} \frac{y_{2}}{y_{2}^{2}+x_{2}^{2}} P(\varepsilon) d x_{1} d x_{2} d y_{1} d y_{2} d \varepsilon$

Поскольку при $V_{1,2} \rightarrow+0$ функция $\eta$ близка к (17), можно считать, что в области, где подынтегральное выражение существенно отлично от нуля, выполняются следующие оценки: $y_{1} \approx V_{1}, y_{2} \approx-V_{2}$, причем точность оценок увеличивается по мере стремления $V_{1,2}$ к нулю. В этом пределе $y_{2} /\left(y_{2}^{2}+x_{2}^{2}\right) \rightarrow-\pi \delta\left(x_{2}\right)$, а $y_{1} \rightarrow+0$. Имея это в виду, можно выполнить интегрирование по $x_{2}$, после чего подставить вместо функции $\eta$ ее предельное выражение (17) и окончательно получить следующее соотношение:

$$
\left\langle y_{2} x_{1}\right\rangle=\pi \int \rho\left(U_{1}-\varepsilon+x, U_{2}-\varepsilon\right) P(\varepsilon) \frac{d x}{x} d \varepsilon
$$

причем интеграл здесь понимается в смысле главного значения. Аналогичные вычисления можно проделать для $\left\langle y_{1} x_{2}\right\rangle,\left\langle y_{1} y_{2}\right\rangle$ и $\left\langle x_{1} x_{2}\right\rangle$.

Наконец, отметим еще одно важное обстоятельство. Из выражения (15) видно, что если $\left\langle y_{2} x_{1}\right\rangle$ не имеет сингулярности при $U_{2}-U_{1} \equiv \omega=0, \operatorname{so~} \lim _{t \rightarrow \infty} \Delta_{\left\langle y_{2} x_{1}\right\rangle}=0$. Поэтому ненулевое значение $D$ связано с появлением сингулярности величины $\left\langle y_{2} x_{1}\right\rangle$ при $\omega=0$. Таким образом, для вычисления величины $D$ достаточно решить уравнение (18) при малой разности $\omega$, выделить в этом решении сингулярную часть и с ее помощью определить предельное при $t \rightarrow \infty$ поведение интеграла в (15). Этому посвящены следующие разделы. 


\section{3. БИНАРНЫЙ БЕСПОРЯДОК}

В случае бинарного беспорядка, о котором шла речь в первом разделе, плотность распределения атомных расщеплений имеет вид

$$
P(y)=(1-c) \delta(y)+c \delta(y-\varepsilon), \quad 0<c<1,
$$

а уравнение (18) записывается следующим образом:

$$
x_{1}^{2} x_{2}^{2} \rho\left(x_{1}, x_{2}\right)=(1-c) \rho\left(U_{1}-\frac{1}{x_{1}}, U_{2}-\frac{1}{x_{2}}\right)+c \rho\left(U_{1}-\varepsilon-\frac{1}{x_{1}}, U_{2}-\varepsilon-\frac{1}{x_{2}}\right) .
$$

Представим функцию $\rho$ в виде ряда по степеням $c$ :

$$
\rho=\sum_{n=0}^{\infty} c^{n} \rho_{n} .
$$

Подставляя этот ряд в (23) и приравнивая коэффициенты при одинаковых степенях $c$, получаем:

$$
\begin{array}{ll}
c^{0}: & \rho_{0}\left(x_{1}, x_{2}\right) x_{1}^{2} x_{2}^{2}=\rho_{0}\left(U_{1}-\frac{1}{x_{1}}, U_{2}-\frac{1}{x_{2}}\right) \\
c^{1}: & \rho_{1}\left(x_{1}, x_{2}\right) x_{1}^{2} x_{2}^{2}=\rho_{1}\left(U_{1}-\frac{1}{x_{1}}, U_{2}-\frac{1}{x_{2}}\right)+ \\
& +\rho_{0}\left(U_{1}-\varepsilon-\frac{1}{x_{1}}, U_{2}-\varepsilon-\frac{1}{x_{2}}\right)-\rho_{0}\left(U_{1}-\frac{1}{x_{1}}, U_{2}-\frac{1}{x_{2}}\right)
\end{array}
$$

и т. д.

Для расчета величины $D$ с точностью до членов порядка $c^{2}$ достаточно рассчитать с этой точностью среднее (21). Подставляя в (21) функции (22) и (24), получим

$$
\left\langle y_{2} x_{1}\right\rangle=\pi \int\left\{(1-c) \rho_{0}\left(U_{1}+x, U_{2}\right)+c \rho_{0}\left(U_{1}-\varepsilon+x, U_{2}-\varepsilon\right)+c \rho_{1}\left(U_{1}+x, U_{2}\right)\right\} \frac{d x}{x}+O\left(c^{2}\right) .
$$

Первое слагаемое в фигурных скобках с точностью до множителя $1-c$ дает значение среднего $\left\langle y_{2} x_{1}\right\rangle$ для полностью упорядоченной цепочки, когда $D=0$, и интереса для нас не представляет. Таким образом, для интересующей нас сингулярной части $\left\langle y_{2} x_{1}\right\rangle$ (обозначим ее символом sing) и предельного при $t \rightarrow \infty$ значения $\Delta_{\left\langle y_{2} x_{1}\right\rangle}$ получаем следующие выражения:

$$
\begin{gathered}
\operatorname{sing}\left\langle y_{2} x_{1}\right\rangle=c \pi \int\left\{\rho_{0}\left(U_{1}-\varepsilon+x, U_{2}-\varepsilon\right)+\rho_{1}\left(U_{1}+x, U_{2}\right)\right\} \frac{d x}{x}+O\left(c^{2}\right), \\
\lim _{t \rightarrow \infty} \Delta_{\left\langle y_{2} x_{1}\right\rangle}=\frac{i}{4 \pi^{2}} \lim _{t \rightarrow \infty} \int e^{i\left(U_{1}-U_{2}\right) t} \operatorname{sing}\left\langle y_{2} x_{1}\right\rangle d U_{1} d U_{2} .
\end{gathered}
$$

Чтобы найти входящие в (28) функции $\rho_{0}$ и $\rho_{1}$, необходимо решить уравнения $(25)$ и (26). Мы начнем анализ этих уравнений с особенно важного случая $\left|U_{1,2}\right|<2$, т. е. при условии, что энергии обеих функций Грина попадают в спектр гамильтониана (1) при $\varepsilon_{r}=0$. 
3.1. Вклад области $\left|U_{1,2}\right|<2$. Прямая подстановка показывает, что при $\left|U_{1,2}\right|<2$ решение уравнения $(25)$ для $\rho_{0}$ может быть представлено в явном виде

$$
\rho_{0}\left(x_{1}, x_{2}\right)=\mathcal{L}_{U_{1}}\left(x_{1}\right) \mathcal{L}_{U_{2}}\left(x_{2}\right)
$$

где $\mathcal{L}_{U}(x)$ - лоренциан,

$$
\mathcal{L}_{U}(x)=\frac{\sqrt{4-U^{2}}}{2 \pi} \frac{1}{x^{2}-U x+1} .
$$

Перейдем теперь к уравнению (26), решение которого при $\left|U_{1,2}\right|<2$ будем строить в виде разложения по предложенной автором в работе [9] системе специальных функций. Приведем полученные в [9] результаты. Введем зависящий от параметра $U,|U|<2$, линейный оператор $\mathcal{H}_{U}$, осуществляющий в функции, на которую он действует, замену переменной $x \mapsto U-1 / x$ с последующим делением результата на $x^{2}$ :

$$
\mathcal{H}_{U} f(x) \equiv \frac{1}{x^{2}} f\left(U-\frac{1}{x}\right)
$$

Как показано в [9], собственные функции $\sigma_{U}^{(n)}(x)$ и собственные значения $\lambda_{n}$ этого оператора нумеруются целым числом $n$ и определяются соотношениями

$$
\begin{gathered}
\sigma_{U}^{(n)}(x)=\mathcal{L}_{U}(x)\left[\frac{R^{*}-x}{R-x}\right]^{n} \equiv \mathcal{L}_{U}(x) G^{n}(x), \\
\lambda_{n}=\left(\frac{U+i \sqrt{4-U^{2}}}{U-i \sqrt{4-U^{2}}}\right)^{n}, \quad\left|\lambda_{n}\right|=1
\end{gathered}
$$

где

$$
R=\frac{U+i \sqrt{4-U^{2}}}{2}, \quad R^{*}=\frac{U-i \sqrt{4-U^{2}}}{2}, \quad R R^{*}=1 .
$$

Отображение, осуществляемое оператором $\mathcal{H}_{U}$, фигурирует в уравнениях (25), (26), поэтому решения этих уравнений будем искать в виде ряда по функциям (32). Для этого используем полученные в [9] правила разложения произвольной функции $f(x)$ :

$$
f(x)=\sum_{n=-\infty}^{+\infty} A_{n} \sigma_{U}^{(n)}(x)
$$

где коэффициенты $A_{n}$ определяются формулами

$$
A_{n}=\int \frac{f(x)}{G^{n}(x)} d x .
$$

Представим $\rho_{1}\left(x_{1}, x_{2}\right)$ в виде ряда по функциям (32):

$$
\rho_{1}\left(x_{1}, x_{2}\right)=\sum_{|n|+|m| \neq 0} C_{n m} \sigma_{U_{1}}^{(n)}\left(x_{1}\right) \sigma_{U_{2}}^{(m)}\left(x_{2}\right) .
$$


Подставляя этот ряд в уравнение (26) и пользуясь свойствами функций (32), получим

$$
\begin{aligned}
& \sum_{|n|+|m| \neq 0} C_{n m} \sigma_{U_{1}}^{(n)}\left(x_{1}\right) \sigma_{U_{2}}^{(m)}\left(x_{2}\right)\left[1-\lambda_{n}\left(U_{1}\right) \lambda_{m}\left(U_{2}\right)\right]= \\
& =\frac{\rho_{0}\left(U_{1}-\varepsilon-1 / x_{1}, U_{2}-\varepsilon-1 / x_{2}\right)}{x_{1}^{2} x_{2}^{2}}-\mathcal{L}_{U_{1}}\left(x_{1}\right) \mathcal{L}_{U_{2}}\left(x_{2}\right) .
\end{aligned}
$$

Разлагая с помощью (33) и (34) правую часть этого уравнения по функциям (32), получим для коэффициентов разложения $\rho_{1}$ следующие выражения:

$$
C_{n m}=\frac{J_{n}\left(U_{1}, \varepsilon\right) J_{m}\left(U_{2}, \varepsilon\right)}{1-\lambda_{n}\left(U_{1}\right) \lambda_{m}\left(U_{2}\right)}
$$

где функции $J_{n}(U, \varepsilon)$ определяются как

$$
J_{n}(U, \varepsilon) \equiv \int \frac{\mathcal{L}_{U}(U-\varepsilon-1 / x)}{G^{n}(x)} \frac{d x}{x^{2}}=\lambda_{n}(U) \int \frac{\mathcal{L}_{U}(x-\varepsilon)}{G^{n}(x)} d x
$$

мы здесь воспользовались равенством $G(1 /(U-\theta))=G(\theta) / \lambda_{1}[9]$. Имеют место соотношения

$$
\begin{aligned}
& J_{n}(U, 0)=0 \quad \text { при } \quad n \neq 0, \\
& J_{n}(U, \varepsilon)=J_{-n}^{*}(U, \varepsilon) \Rightarrow\left|J_{n}(U, \varepsilon)\right|^{2}=\left|J_{-n}^{*}(U, \varepsilon)\right|^{2} .
\end{aligned}
$$

Таким образом, решение уравнения (26) имеет вид

$$
\rho_{1}\left(x_{1}, x_{2}\right)=\sum_{|n|+|m| \neq 0} \frac{J_{n}\left(U_{1}, \varepsilon\right) J_{m}\left(U_{2}, \varepsilon\right)}{1-\lambda_{n}\left(U_{1}\right) \lambda_{m}\left(U_{2}\right)} \sigma_{U_{1}}^{(n)}\left(x_{1}\right) \sigma_{U_{2}}^{(m)}\left(x_{2}\right) .
$$

Как было отмечено выше, для вычисления величины $D$ нам необходима только сингулярная при $\omega=U_{2}-U_{1} \approx 0$ часть этого выражения. Нетрудно видеть, что особенность при $\omega=0$ имеют только те члены суммы (40), у которых $m=-n$. Сделаем следующую замену символов: $U_{1}=U, U_{2}=U+\omega$ и запишем выражение для знаменателя этих членов с точностью до членов порядка $\omega^{2}$ :

$$
1-\lambda_{n}(U) \lambda_{-n}(U+\omega)=-\frac{2 i n \omega}{\sqrt{4-U^{2}}}+O\left(\omega^{2}\right) .
$$

Принимая это во внимание, получаем для сингулярной части решения (40)

$$
\operatorname{sing} \rho_{1}\left(x_{1}, x_{2}\right)=\frac{i \sqrt{4-U^{2}}}{2 \omega} \sum_{n \neq 0} \frac{\left|J_{n}(U, \varepsilon)\right|^{2}}{n} \sigma_{U}^{(n)}\left(x_{1}\right) \sigma_{U}^{-n}\left(x_{2}\right) .
$$

Обратимся теперь к формуле для $\operatorname{sing}\left\langle y_{2} x_{1}\right\rangle$ в (28). Заметим, что первое слагаемое под интегралом при $\left|U_{1,2}\right|<2$ не вносит вклад в силу регулярности функции $\rho_{0}(29)$ при $\omega=0$. Поэтому нам нужно рассмотреть только вклад второго слагаемого, 
зависящего от функции $\rho_{1}$ :

$$
\begin{aligned}
\operatorname{sing}\left\langle y_{2} x_{1}\right\rangle & =\operatorname{sing}\left(\pi c \int \rho_{1}(U+x, U+\omega) \frac{d x}{x}\right)= \\
& =i \pi c \frac{\sqrt{4-U^{2}}}{2 \omega} \sum_{n \neq 0} \frac{\left|J_{n}(U, \varepsilon)\right|^{2}}{n} \sigma_{U}^{-n}(U) \int \sigma_{U}^{(n)}(U+x) \frac{d x}{x}= \\
& =i c \frac{4-U^{2}}{4 \omega} \sum_{n \neq 0} \frac{\left|J_{n}(U, \varepsilon)\right|^{2}}{n} \int x \sigma_{U}^{(n)}(x) d x .
\end{aligned}
$$

При преобразовании подынтегрального выражения мы сделали замену переменной $x \mapsto-1 / x$ и воспользовались тем, что функции $\sigma_{U}^{(n)}(x)$ являются собственными для оператора $\mathcal{H}_{U}(31)$. Теперь можно вычислить предельное при $t \rightarrow \infty$ поведение вклада (15):

$$
\begin{aligned}
\left.\lim _{t \rightarrow \infty} \Delta_{\left\langle y_{2} x_{1}\right\rangle}\right|_{\left|U_{1,2}\right|<2}= & -\frac{c}{16 \pi^{2}} \lim _{t \rightarrow \infty} \int \frac{e^{i \omega t}}{\omega} d \omega \times \\
& \times \int_{-2}^{2}\left(4-U^{2}\right) \sum_{n \neq 0} \frac{\left|J_{n}(U, \varepsilon)\right|^{2}}{n} d U \int x \sigma_{U}^{(n)}(x) d x .
\end{aligned}
$$

Предел при $t \rightarrow \infty$ первого интеграла не зависит от области интегрирования по $\omega:$

$$
\lim _{t \rightarrow \infty} \int \frac{e^{i \omega t}}{\omega} d \omega=\int_{-\infty}^{\infty} \frac{e^{i x}}{x} d x=i \pi
$$

Вычисление первого момента функции $\sigma^{(n)}(x)$ и интеграла $(38)$ приводит к следующим выражениям:

$$
\int x \sigma_{U}^{(n)}(x) d x=\frac{i}{2} \frac{n}{|n|} \sqrt{4-U^{2}}, \quad\left|J_{n}(U, \varepsilon)\right|^{2}=\left(\frac{\varepsilon^{2}}{\varepsilon^{2}+4-U^{2}}\right)^{n} .
$$

Таким образом, формула (44) может быть преобразована так:

$$
\left.\lim _{t \rightarrow \infty} \Delta_{\left\langle y_{2} x_{1}\right\rangle}\right|_{\left|U_{1,2}\right|<2}=\frac{c}{16 \pi} \int_{-2}^{2}\left(4-U^{2}\right)^{3 / 2} \sum_{n=1}^{\infty} \frac{1}{n}\left(\frac{\varepsilon^{2}}{\varepsilon^{2}+4-U^{2}}\right)^{n} d U+O\left(c^{2}\right) .
$$

Пользуясь тем, что $\sum_{n=1}^{\infty} q^{n} / n=-\ln (1-q)$, получаем

$$
\left.\lim _{t \rightarrow \infty} \Delta_{\left\langle y_{2} x_{1}\right\rangle}\right|_{\left|U_{1,2}\right|<2}=\frac{c}{16 \pi} \int_{-2}^{2}\left(4-U^{2}\right)^{3 / 2} \ln \left(\frac{\varepsilon^{2}}{4-U^{2}}+1\right) d U+O\left(c^{2}\right) .
$$

3.2. Вклад области $\left|U_{1,2}\right|>2$. Вернемся к формулам (28) и рассмотрим, какой вклад в фигурирующий в них интеграл вносит область $\left|U_{1,2}\right|>2$. В приложении показано, что в этом случае зависящее от $\rho_{1}$ второе слагаемое в фигурных скобках в (28) роли не играет. Таким образом, весь вклад области $\left|U_{1,2}\right|>2$ связан с первым 
слагаемым, зависящим от $\rho_{0}$. Поскольку выражение для КФГ упорядоченной цепочки известно ${ }^{1)}$, решение уравнения $(25)$ для невозмущенной функции совместной плотности вероятности $\rho_{0}$ при $\left|U_{1,2}\right|>2$ несложно угадать:

$$
\rho_{0}\left(x_{1}, x_{2}\right)=\delta\left[x_{1}-\frac{U_{1}-\operatorname{sign}\left(U_{1}\right) \sqrt{U_{1}^{2}-4}}{2}\right] \delta\left[x_{2}-\frac{U_{2}-\operatorname{sign}\left(U_{2}\right) \sqrt{U_{2}^{2}-4}}{2}\right] .
$$

Рассчитаем теперь вклад связанного с $\rho_{0}$ первого слагаемого в фигурных скобках в (28) (обозначим его $\left.I_{1}\right)$ :

$$
\begin{aligned}
I_{1}= & \frac{i c}{4 \pi} \int e^{i\left(U_{1}-U_{2}\right) t} \rho_{0}\left(U_{1}-\varepsilon+x, U_{2}-\varepsilon\right) \frac{d x d U_{1} d U_{2}}{x}= \\
= & \frac{i c}{4 \pi} \int e^{i\left(U_{1}-U_{2}\right) t} \delta\left[x-\varepsilon+\frac{U_{1}+\operatorname{sign}\left(U_{1}\right) \sqrt{U_{1}^{2}-4}}{2}\right] \times \\
& \times \delta\left[\frac{U_{2}+\operatorname{sign}\left(U_{2}\right) \sqrt{U_{2}^{2}-4}}{2}-\varepsilon\right] \frac{d x d U_{1} d U_{2}}{x} .
\end{aligned}
$$

Здесь интегралы по $U_{1,2}$ берутся по области $\left|U_{1,2}\right|>2$. Интегрируя (50) по $x$, получаем

$$
I_{1}=\frac{i c}{4 \pi} \int \frac{e^{i\left(U_{1}-U_{2}\right) t} d U_{1} d U_{2}}{\varepsilon-\left[U_{1}+\operatorname{sign}\left(U_{1}\right) \sqrt{U_{1}^{2}-4}\right] / 2} \delta\left[\frac{U_{2}+\operatorname{sign}\left(U_{2}\right) \sqrt{U_{2}^{2}-4}}{2}-\varepsilon\right] .
$$

Ясно, что результат не зависит от знака $\varepsilon$, и ниже для определенности будем считать, что $\varepsilon>0$. В этом случае второй сомножитель под интегралом в (51) равен нулю при $U_{2}<-2$, а для $0<\varepsilon<1$ равен нулю тождественно. Следовательно, рассчитываемый вклад отличен от нуля только при $|\varepsilon|>1$. Подынтегральные функции в (51) имеют особенность типа $\delta$-функции при интегрировании по $U_{2}$ и особенность типа полюса при интегрировании по $U_{1}$. Для вычисления этих интегралов (при $\left.t \rightarrow \infty\right)$ примем во внимание, что фигурирующая в них функция

$$
\Phi(U) \equiv \frac{U+\sqrt{U^{2}-4}}{2}-\varepsilon
$$

обращается в нуль при

$$
U=U_{0} \equiv \frac{\varepsilon^{2}+1}{\varepsilon}
$$

и может быть разложена в ряд при $U \approx U_{0}$ :

$$
\Phi(U) \approx\left(U-U_{0}\right) \frac{\varepsilon^{2}}{\varepsilon^{2}-1} .
$$

Используя эту формулу, вычисляем входящий в (51) интеграл по $U_{2}$ :

$$
\int e^{-i U_{2} t} \delta\left[\frac{U_{2}+\sqrt{U_{2}^{2}-4}}{2}-\varepsilon\right] d U_{2}=\frac{\varepsilon^{2}-1}{\varepsilon^{2}} e^{-i U_{0} t} .
$$

\footnotetext{
1) Оно получается путем решения уравнения (9) при $\widetilde{\gamma}=\gamma, \varepsilon=0$.
} 
Рассмотрим теперь интеграл по $U_{1}$ в $(51)$. Поскольку его надо вычислить при $t \rightarrow \infty$, ненулевым будет только вклад полюса подынтегрального выражения. Опять используя разложение (54), получаем для этого интеграла следующее выражение:

$$
\int_{U_{1}>2} \frac{e^{i U_{1} t} d U_{1}}{\varepsilon-\left[U_{1}+\sqrt{U_{1}^{2}-4}\right] / 2} \approx-\frac{\varepsilon^{2}-1}{\varepsilon^{2}} \int_{-\infty}^{+\infty} \frac{e^{i U_{1} t} d U_{1}}{U_{1}-U_{0}}=-i \pi e^{i U_{0} t} \frac{\varepsilon^{2}-1}{\varepsilon^{2}} .
$$

Здесь приближенное равенство становится точным в пределе $t \rightarrow \infty$, который нас как раз и интересует. Таким образом,

$$
I_{1}=\Theta(|\varepsilon|-1) \frac{c}{4}\left(\frac{\varepsilon^{2}-1}{\varepsilon^{2}}\right)^{2} .
$$

Дополнительное исследование (на котором мы не останавливаемся) показывает, что этот вклад связан с появлением при $|\varepsilon|>1$ краевого состояния, которое имеет энергию $U_{0}$, определямую формулой (53). Чтобы получить окончательную формулу для величины $D$, вклады (57) и (48) следует сложить и в соответствии с (16) учетверить результат. Таким образом, для величины $D$ в рассматриваемом случае бинарного беспорядка получается формула (4). В соответствии с логикой ее вывода и замечанием, приведенным после формулы (15), вид функции “участия" $W(U)$, введенной в первом разделе, определяется выражением (5).

\section{4. ПРОИЗВОЛЬНЫЙ МАЛЫЙ ДИАГОНАЛЬНЫЙ БЕСПОРЯДОК}

Проведенный выше анализ бинарно разупорядоченной цепочки представляет собой последовательную теорию возмущений для статистики опережающей и запаздывающей функций Грина, в которой роль малого параметра играет средняя концентрация $c$ атомов с расщеплением $\varepsilon$. Аналогично может быть построена теория возмущений для цепочки с беспорядком достаточно общего вида, причем параметр малости вводится следующим образом. Пусть функция $p(\varepsilon)$ такова, что $p(\varepsilon)>0$, $\int p(\varepsilon) d \varepsilon=1$. С помощью этой функции построим следующее семейство плотностей распределения атомных расщеплений $P_{\Delta}(\varepsilon)$ :

$$
P_{\Delta}(\varepsilon)=\frac{1}{\Delta} p\left(\frac{\varepsilon}{\Delta}\right), \quad \Delta>0 .
$$

Если определить моменты функции $p(\varepsilon)$ как

$$
M_{n} \equiv \int p(\varepsilon) \varepsilon^{n} d \varepsilon
$$

то моменты функций (58) могут быть выражены через них следующим образом:

$$
\int P_{\Delta}(\varepsilon) \varepsilon^{n} d \varepsilon=\Delta^{n} M_{n}
$$

Ясно, что $\Delta$ есть мера разупорядоченности системы: при $\Delta \rightarrow 0$ система становится упорядоченной. Без потери общности можно считать, что $M_{1}=0$. Таким образом, 
нужно построить теорию возмущений для уравнения $(18)$ при $P(\varepsilon)=P_{\Delta}(\varepsilon)$, где малым параметром является величина $\Delta$.

Будем строить разложение решения (18) по степеням $\Delta$ и проделаем вычисления для особенно важного случая $\left|U_{1,2}\right|<2$. Для этого напишем разложение функции $\rho\left(x_{1}, x_{2}\right)$ в окрестности некоторой точки $x_{10}, x_{20}$ :

$$
\begin{aligned}
\rho\left(x_{1}, x_{2}\right) & =\sum_{n, m=0}^{\infty} \rho_{n m}\left(x_{1}-x_{10}\right)^{n}\left(x_{2}-x_{20}\right)^{m}, \\
\rho_{n m} & \left.\equiv \frac{1}{n ! m !} \frac{\partial^{n+m}}{\partial \theta_{1}^{n} \partial \theta_{2}^{m}} \rho\left(\theta_{1}, \theta_{2}\right)\right|_{\theta_{i}=x_{i 0}} .
\end{aligned}
$$

Применяя это разложение в правой части (18) и полагая $x_{i 0}=U_{i}-1 / x_{i}, i=1,2$, можно получить разложение функции $\rho\left(U_{1}-\varepsilon-1 / x_{1}, U_{2}-\varepsilon-1 / x_{2}\right)$ по степням $\varepsilon$ и выразить правую часть (18) через моменты (59) и степени $\Delta$ :

$$
\begin{aligned}
& \int P_{\Delta}(\varepsilon) \rho\left(U_{1}-\varepsilon-\frac{1}{x_{1}}, U_{2}-\varepsilon-\frac{1}{x_{2}}\right) d \varepsilon= \\
& \quad=\left.\sum_{n, m=0}^{\infty} \frac{(-\Delta)^{n+m} M_{n+m}}{n ! m !} \frac{\partial^{n+m} \rho\left(\theta_{1}, \theta_{2}\right)}{\partial \theta_{1}^{n} \partial \theta_{2}^{m}}\right|_{\theta_{1}=U_{1}-1 / x_{1}} ^{\theta_{2}=U_{2}-1 / x_{2}} .
\end{aligned}
$$

Отсюда следует, что и левая часть (18) (т. е. сама функция $\rho\left(x_{1}, x_{2}\right)$ ) допускает разложение по степеням $\Delta$, которое мы запишем в виде

$$
\rho\left(x_{1}, x_{2}\right) \equiv \sum_{k=0}^{\infty} \Delta^{k} Q_{k}\left(x_{1}, x_{2}\right)
$$

Уравнение (18) позволяет выразить функции $Q_{n}$ через функции $Q_{m}$ при $m<n$. Для этого подставим разложения (62), (63) в (18). Получим

$$
x_{1}^{2} x_{2}^{2} \sum_{k=0}^{\infty} \Delta^{k} Q_{k}\left(x_{1}, x_{2}\right)=\left.\sum_{n, m, k=0}^{\infty} \frac{(-1)^{n+m} \Delta^{n+m+k} M_{n+m}}{n ! m !} \frac{\partial^{n+m}}{\partial \theta_{1}^{n} \partial \theta_{2}^{m}} Q_{k}\left(\theta_{1}, \theta_{2}\right)\right|_{\theta_{i}=U_{i}-1 / x_{i}} .
$$

Приравнивание коэффициентов при $\Delta^{0}$ дает

$$
x_{1}^{2} x_{2}^{2} Q_{0}\left(x_{1}, x_{2}\right)=Q_{0}\left(U_{1}-\frac{1}{x_{1}}, U_{2}-\frac{1}{x_{2}}\right) .
$$

Отсюда аналогично (29) получаем следующее выражение для $Q_{0}\left(x_{1}, x_{2}\right)$ :

$$
Q_{0}\left(x_{1}, x_{2}\right)=\rho_{0}\left(x_{1}, x_{2}\right)=\mathcal{L}_{U_{1}}\left(x_{1}\right) \mathcal{L}_{U_{2}}\left(x_{2}\right) .
$$

Нетрудно убедиться в том, что равенство нулю первого момента $M_{1}=0$ влечет $Q_{1}\left(x_{1}, x_{2}\right)=0$. Приравнивание коэффициентов при $\Delta^{2}$ дает

$$
\begin{aligned}
x_{1}^{2} x_{2}^{2} Q_{2}\left(x_{1}, x_{2}\right)= & Q_{2}\left(U_{1}-\frac{1}{x_{1}}, U_{2}-\frac{1}{x_{2}}\right)+ \\
& +\left.M_{2}\left[\frac{\partial^{2}}{\partial \theta_{1} \partial \theta_{2}}+\frac{1}{2} \frac{\partial^{2}}{\partial \theta_{1}^{2}}+\frac{1}{2} \frac{\partial^{2}}{\partial \theta_{2}^{2}}\right] Q_{0}\left(\theta_{1}, \theta_{2}\right)\right|_{\theta_{i}=U_{i}-1 / x_{i}} .
\end{aligned}
$$


Вычислим теперь с точностью до членов порядка $\Delta^{3}$ величину $\left\langle y_{2} x_{1}\right\rangle$, определяемую формулой $(21)$ при $P(\varepsilon)=P_{\Delta}(\varepsilon)$. Для фигурирующего в $\left\langle y_{2} x_{1}\right\rangle$ интеграла используем разложения (62), (63):

$$
\begin{aligned}
& \int P_{\Delta}(\varepsilon) \rho\left(U_{1}-\varepsilon+x, U_{2}-\varepsilon\right) d \varepsilon= \\
& =\left.\int d \varepsilon P_{\Delta}(\varepsilon) \sum_{n, m, k=0}^{\infty} \frac{(-\varepsilon)^{n+m} \Delta^{k}}{n ! m !} \frac{\partial^{n+m} Q_{k}\left(\theta_{1}, \theta_{2}\right)}{\partial \theta_{1}^{n} \partial \theta_{2}^{m}}\right|_{\theta_{1}=U_{1}+x} ^{\theta_{2}=U_{2}}= \\
& =\left.\sum_{n, m, k=0}^{\infty} \frac{(-1)^{n+m} \Delta^{n+m+k} M_{n+m}}{n ! m !} \frac{\partial^{n+m} Q_{k}\left(\theta_{1}, \theta_{2}\right)}{\partial \theta_{1}^{n} \partial \theta_{2}^{m}}\right|_{\theta_{1}=U_{1}+x} ^{\theta_{2}=U_{2}}=Q_{0}\left(U_{1}+x, U_{2}\right)+ \\
& \quad+\Delta^{2}\left[Q_{2}\left(U_{1}+x, U_{2}\right)+\left.M_{2}\left(\frac{\partial^{2}}{\partial \theta_{1} \partial \theta_{2}}+\frac{1}{2} \frac{\partial^{2}}{\partial \theta_{1}^{2}}+\frac{1}{2} \frac{\partial^{2}}{\partial \theta_{2}^{2}}\right) Q_{0}\left(\theta_{1}, \theta_{2}\right)\right|_{\theta_{1}=U_{1}+x} ^{\theta_{2}=U_{2}}\right]+O\left(\Delta^{3}\right) .
\end{aligned}
$$

Здесь первый член нулевого порядка по $\Delta$ соответствует цепочке без беспорядка и поэтому не дает вклада в интересующую нас величину $D$. Для расчета вклада в величину $D$ первого слагаемого в квадратных скобках (назовем его $A$-вкладом) необходима функция $Q_{2}\left(x_{1}, x_{2}\right)$, которая находится из уравнения (67). Решить это уравнение при $\left|U_{1,2}\right|<2$ можно так же, как и уравнение $(26)$, и решение сводится к следующему переопределению величин $J_{n}(U)$ :

$$
\left.J_{n}(U) \equiv \int \frac{d x}{x^{2} G^{n}(x)} \frac{\partial \mathcal{L}_{U}(\theta)}{\partial \theta}\right|_{\theta=U-1 / x}, \quad J_{n}(U)=J_{-n}^{*}(U) .
$$

Делая в этих интегралах замену переменной $\theta=U-1 / x$ и пользуясь тем, что функция $G(\theta)(32)$ удовлетворяет равенству $G(1 /(U-\theta))=G(\theta) / \lambda_{1}[9]$, получаем

$$
J_{n}(U)=\lambda_{n} \int \frac{d \mathcal{L}_{U}(x)}{d x} \frac{d x}{G^{n}(x)} .
$$

Вычисление этих интегралов при различных $n$ показывает, что отличными от нуля будут только $J_{ \pm 1}(U)$ :

$$
J_{ \pm 1}(U)=\mp \frac{i \lambda_{1}}{\sqrt{4-U^{2}}}, \quad\left|J_{ \pm 1}(U)\right|^{2}=\frac{1}{4-U^{2}} .
$$

Имея это в виду, уже нетрудно убедиться в том, что $A$-вклад из (68) при $\left|U_{1,2}\right|<2$ будет таким:

$$
\left.\Delta_{\left\langle y_{2} x_{1}\right\rangle}(\infty)\right|_{A \text {-вклад }}=\frac{\Delta^{2} M_{2}}{16 \pi} \int_{-2}^{2} \sqrt{4-U^{2}} d U=\frac{\Delta^{2} M_{2}}{8} .
$$

В случае $U_{1,2}>2$ анализ уравнения (67), аналогичный приведенному в приложении, показывает, что $A$-вклад равен нулю.

Рассмотрим теперь вклад в величину $D$ второго слагаемого в квадратных скобках в (68) - назовем его $B$-вкладом. Для этой величины область $U_{1,2}<2$ не играет 
роли при вычислении предельного при $t \rightarrow \infty$ поведения $\Delta_{\left\langle y_{2} x_{1}\right\rangle}$, поскольку в этом случае под интегралами стоят функции, не имеющие особенностей при $U_{1}=U_{2}$. При $\left|U_{1,2}\right|>2$ В-вклад из (68) имеет вид

$$
\begin{aligned}
\left.\Delta_{\left\langle y_{2} x_{1}\right\rangle}\right|_{\text {В-вклад }}= & M_{2} \Delta^{2} \pi \int \frac{d x}{x} \frac{d}{d x} \delta\left(x+\frac{U_{1}+\operatorname{sign}\left(U_{1}\right) \sqrt{U_{1}^{2}-4}}{2}\right) \times \\
& \times\left.\frac{d}{d y} \delta\left(y+\frac{U_{2}+\operatorname{sign}\left(U_{2}\right) \sqrt{U_{2}^{2}-4}}{2}\right) e^{i\left(U_{1}-U_{2}\right) t}\right|_{y=0} ^{t \rightarrow \infty}+\cdots .
\end{aligned}
$$

Для сокращения записи мы привели здесь только член со смешанными производными, оставшиеся члены анализируются так же. Поскольку аргумент второй $\delta$-функции нигде не обращается в нуль (при $\left|U_{2}\right|>2$ ), мы приходим к заключению, что $B$-вклад равен нулю и, учетверяя результат (72), получаем формулы (6).

\section{5. ЧИСЛЕННЫЙ ЭКСПЕРИМЕНТ И ЗАКЛЮЧЕНИЕ}

Полученные выше формулы (4)-(6) могут быть проверены с помощью расчетов величины $D$ и функции $W(U)$ по формулам $(2)$ и $(3)$, в которых собственные векторы $\boldsymbol{\Psi}^{\lambda}$ и собственные энергии $E_{\lambda}$ получены прямой компьютерной диагонализацией гамильтониана (1).

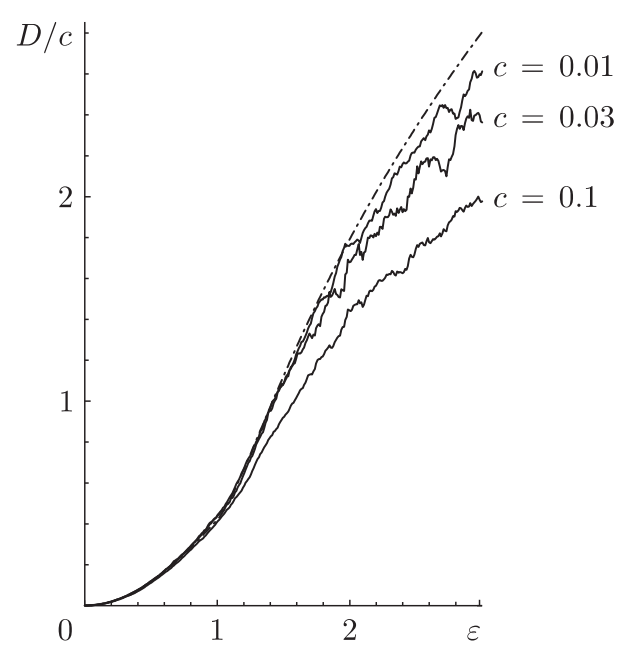

a

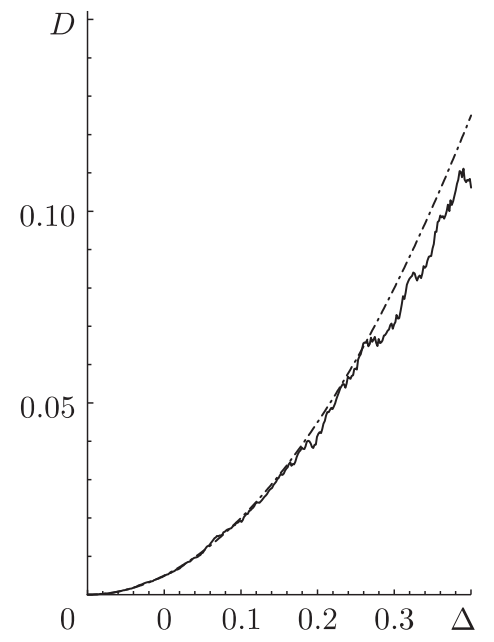

б

Рис. 1. Случай бинарного беспорядка (а): сплошные кривые - зависимости величины $D / c$ от энергии дефектов $\varepsilon$, полученные численно при разных концентрациях дефектов $c=0.01,0.03,0.1$, штрихпунктир - теоретическая зависимость. Случай равномерного беспорядка (б): зависимость предельной при $t \rightarrow \infty$ плотности возбуждения $D$ на крайнем узле от величины беспорядка $\Delta$, рассчитанная численно (сплошная кривая) и по формуле (6) (штрихпунктир). 

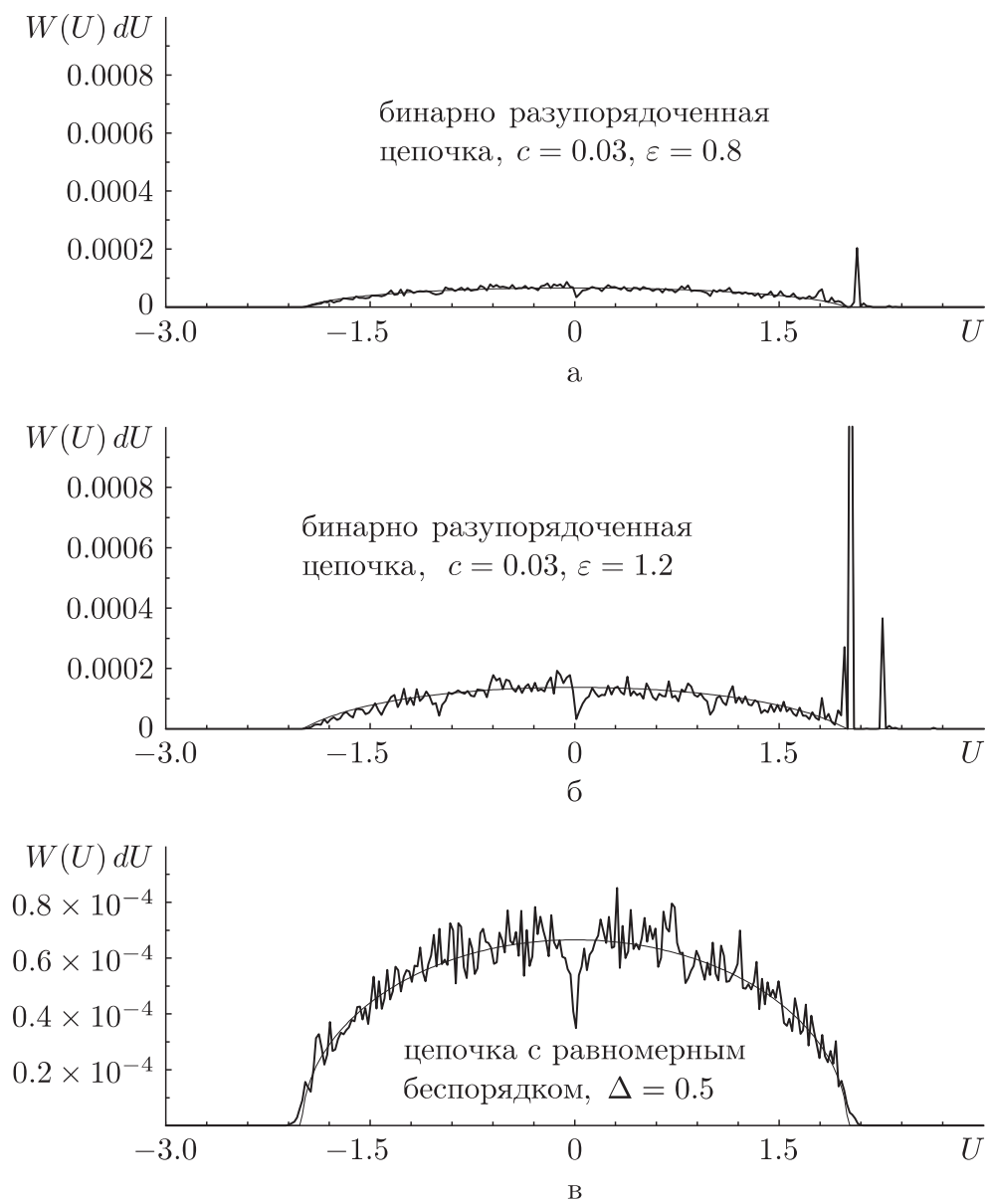

Рис. 2. Функция “участия”. Сравнение теории (гладкие кривые) с компьютерным моделированием (зашумленные кривые). Для бинарно разупорядоченной цепочки видно появление особенности, связанной с краевым состоянием: $\varepsilon=0.8<1$ (а), особенность выражена слабо; $\varepsilon=1.2>1$ (б), появляется высокий узкий пик функции “участия"; функция “участия” для цепочки с равномерным беспорядком при $\Delta=0.5$ (в). Во всех случаях $d U=1 / 50$.

Ниже мы приводим результаты такой проверки, причем зашумленные кривые получены численно, а гладкие - с помощью формул (4)-(6). На рис. 1а для бинарно разупорядоченной цепочки представлены зависимости величины $D / c$ от энергии дефекта $\varepsilon$, полученные численно для разных концентраций дефектов $c$, и соответствующая теоретическая зависимость (4), показанная штрихпунктиром. Видно, что если $D / c$ не зависит от $c$, то формула (4) полностью соответствует численным кривым, на которых хорошо заметен изгиб при $\varepsilon=1$, описываемый неаналитическим членом в (4). На рис. 1 представлена зависимость величины $D$ от степени разупорядоченности $\Delta$ для случая равномерного беспорядка, когда функция распределения 
атомных расщеплений берется в виде (58), причем $p(x)=\Theta(0.5-|x|)$. Видно, что формула (6) (штрихпунктир) хорошо описывает численный эксперимент даже при относительно большом беспорядке.

На рис. 2 представлены зависимости функций участия $W(U) d U$ от энергии, полученные численно с помощью формулы (3) и рассчитанные теоретически по формулам (5) (бинарный беспорядок) и (6) (равномерный беспорядок). Рис. 2а, 26 относятся к бинарно разупорядоченной системе с концентрацией дефектов $c=0.03$, причем рис. 2а соответствует энергии дефекта $\varepsilon=0.8$, а рис. $2 б-$ энергии $\varepsilon=1.2$. Из этих рисунков видно, что при $\varepsilon>1$ функция "участия" $W(U)$ демонстрирует резкий максимум при $U=U_{0}(53)$. Рис. 2в соответствует цепочке с довольно большим $(\Delta=0.5)$ равномерным беспорядком. Видно, что и в этом случае имеется хорошее согласие теории и численного эксперимента, однако для описания заметного провала в центре "экспериментальной" зависимости требуется, по-видимому, учет поправок более высокого порядка чем $\Delta^{2}$. При численном моделировании не применялось никакой подгонки.

Заметим, что для получения численных зависимостей, приведенных на рис. 1, можно использовать небольшие матрицы $(N \sim 100)$ и вычислять $D$ как $\left|\Psi_{N}(t)\right|^{2}$ при $t=50 \div 100$, проверяя ${ }^{2)}$ отсутствие зависимости результатов от $t$ и $N$. Для получения численных зависимостей на рис. 2 мы использовали матрицы размером $2000 \times 2000$ с последующим усреднением по 1000 реализаций беспорядка.

В заключение сделаем следующее замечание. Появление неаналитичности в зависимости $D$ от энергий дефекта (4) свидетельствует о невозможности анализа бинарно разупорядоченной цепочки в рамках обычной квантово-механической теории возмущений при произвольной энергии дефектов $\varepsilon$. С другой стороны, простой аналитический вид формул (6) для слабо разупорядоченной системы дает основания думать, что для этого случая такой анализ возможен. Предложенная схема теории возмущений для статистики функций Грина позволяет рассмотреть эти две разнородные задачи с единой точки зрения.

\section{ПРИЛОЖКЕНИЕ}

Чтобы определить роль связанного с $\rho_{1}$ второго слагаемого в (28) при $\left|U_{1,2}\right|>2$, необходимо получить решение уравнения (26) для этой спектральной области. Будем для определенности считать, что $U_{1,2}>2$ и введем следующие величины:

$$
\gamma_{U} \equiv \frac{U-\sqrt{U^{2}-4}}{2}, \quad \gamma_{U}^{\prime}=\gamma_{U}^{\prime}(\varepsilon) \equiv \frac{1}{U-\gamma_{U}-\varepsilon},
$$

причем $\gamma_{U}$ является КФГ цепочки без беспорядка. В рассматриваемом случае $U_{1,2}>2$ решение $(25)$ дает для функции $\rho_{0}$ следующее выражение:

$$
\rho_{0}\left(x_{1}, x_{2}\right)=\delta\left(x_{1}-\gamma_{U_{1}}\right) \delta\left(x_{2}-\gamma_{U_{2}}\right) .
$$

2) При небольших $N$ функция $\left|\Psi_{N}(t)\right|^{2}$ обнаруживает квазициклическое поведение, поэтому величину $t$ следует брать меньшей времени цикла. 
Тогда (26) можно переписать так:

$$
\begin{aligned}
& \rho_{1}\left(x_{1}, x_{2}\right)-\frac{\rho_{1}\left(U_{1}-1 / x_{1}, U_{2}-1 / x_{2}\right)}{x_{1}^{2} x_{2}^{2}}= \\
& \quad=\delta\left(x_{1}-\gamma_{U_{1}}^{\prime}\right) \delta\left(x_{2}-\gamma_{U_{2}}^{\prime}\right)-\delta\left(x_{1}-\gamma_{U_{1}}\right) \delta\left(x_{2}-\gamma_{U_{2}}\right) .
\end{aligned}
$$

Непосредственной подстановкой проверяется, что решение уравнения (76) имеет вид

$$
\rho_{1}\left(x_{1}, x_{2}\right)=\lim _{M \rightarrow \infty} \sum_{i=1}^{M}\left\{\delta\left(x_{1}-\theta_{i}\left(U_{1}\right)\right) \delta\left(x_{2}-\theta_{i}\left(U_{2}\right)\right)-\delta\left(x_{1}-\gamma_{U_{1}}\right) \delta\left(x_{2}-\gamma_{U_{2}}\right)\right\},
$$

где величины $\theta_{i}(U)$ определяются рекуррентными соотношениями

$$
\theta_{1}(U)=\gamma_{U}^{\prime}, \quad \theta_{n+1}(U)=\frac{1}{U-\theta_{n}(U)}, \quad n=1,2, \ldots, M-1 .
$$

Для входящей в $(28)$ функции $\rho_{1}\left(U_{1}+x, U_{2}\right)$, пользуясь $(76)$, можно написать выражение

$$
\begin{aligned}
\rho_{1}\left(U_{1}+x, U_{2}\right)= & \lim _{y \rightarrow \infty}\left(\frac{y}{x}\right)^{2}\left[\rho_{1}\left(-\frac{1}{x}, y\right)-\right. \\
& \left.-\delta\left(\frac{1}{x}+\gamma_{U_{1}}^{\prime}\right) \delta\left(y-\gamma_{U_{2}}^{\prime}\right)+\delta\left(\frac{1}{x}+\gamma_{U_{1}}\right) \delta\left(y-\gamma_{U_{2}}\right)\right] .
\end{aligned}
$$

Очевидно, два последних члена с $\delta$-функциями имеют нулевой предел при $y \rightarrow \infty$. Подстановка в это выражение функции $\rho_{1}$ в виде (77) показывает, что предел первого члена также равен нулю. Таким образом, вклад второго слагаемого в (28) при $\left|U_{1,2}\right|>2$ оказывается нулевым.

\section{Список литературы}

[1] И. М. Лифшиц, С.А. Гредескул, Л.А. Пастур, Введение в теорию неупорядоченных систем, Наука, М., 1982.

[2] P.W. Anderson, Phys. Rev., 109:5 (1958), 1492-1505.

[3] Л.А. Пастур, O спектре случайных якобиевых матрии и оператора Шредингера на всей оси со случайным потенииалом, Препринт, ФТИНТ АН УССР, Харьков, 1974; L. A. Pastur, Comm. Math. Phys., 75:2 (1980), 179-196.

[4] И. Я. Гольдшейд, С. А. Молчанов, Л. А. Пастур, Функи. анализ и его прил., 11:1 (1977), $1-10$.

[5] М. В. Белоусов, Д. Е. Погарев, Писъма в ЖЖЭТФ, 36:5 (1982), 152-155.

[6] М. В. Белоусов, Б. Е. Вольф, Е. А. Иванова, Писъма в ЖЭЭТФ, 38:8 (1983), 376-379.

[7] A. V. Malyshev, V. A. Malyshev, F. Domínguez-Adame, Phys. Rev. B, 70:17 (2004), 172202; arXiv: cond-mat/0303092.

[8] F. J. Dyson, Phys. Rev., 92:6 (1953), 1331-1338.

[9] G. G. Kozlov, Spectrum and eigen functions of the operator $H_{U} f(x) \equiv f(U-1 / x) / x^{2}$ and strange attractor's density for the mapping $x_{n+1}=1 /\left(U-x_{n}\right)$, arXiv:0803.1920. 